\title{
Numerical Simulation of the Global Star Formation Pattern in the LMC
}

\section{L.T. Gardiner}

Dept. of International Education, Sun Moon University, Rep. of Korea

C. Turfus

Dept. of Mathematics, Sun Moon University, Rep. of Korea

\begin{abstract}
We have constructed a dynamical model of the LMC to examine the effects of an off-center bar perturbation on the global distribution of the gas and star formation activity. We find that a weak rotating bar potential, whose center is displaced by $0.6 \mathrm{kpc}$ from the disk center, gives rise to an asymmetric spiral structure which mimics the observed chains of recent star formation as well as activity in the bar region.
\end{abstract}

\section{Numerical Model}

Recently, Dottori et al. (1996) proposed that the large-scale gravitational potential due to an off-centered bar has induced the formation of spiral features in the distribution of young star clusters in the LMC. We have conducted numerical simulations to examine this scenario in greater detail. Our numerical model of the LMC was based on the $N$-body/cellular automaton scheme of Gardiner et al. (1998a), which incorporates star formation based on local gravitational instability and propagation within a cloud complex. We simulated the gas disk of the LMC with 7000 particles, representing a total gas mass of $4.2 \times 10^{8} M_{\odot}$, initially distributed uniformly on the surface of a disk of radius $3.5 \mathrm{kpc}$. The particles were subjected to their mutual gravitational forces, as well as to external forces due to the dynamical mass of the SMC associated with the stellar component. These external forces were considered to arise from the superposition of an axisymmetric potential and a weak rotating bar-like perturbation whose center was offset from the disk center by $0.6 \mathrm{kpc}$. The bar was assumed to spin about its own axis at the same rate as it rotates about the disk center with a pattern speed of $\Omega_{b}=50 \mathrm{~km} / \mathrm{s} / \mathrm{kpc}$.

\section{Results}

Figure 1 shows the distribution of star particles up to $30 \mathrm{Myr}$ old produced by the simulation at $T=300 \mathrm{Myr}$. The simulation produces a trailing spiral arm pattern which corresponds to the spiral arms A and B defined by Smith et al. (1987). The pattern also resembles the distribution of young supergiant stars found by Grebel \& Brandner (1998, these proceedings). Although the 


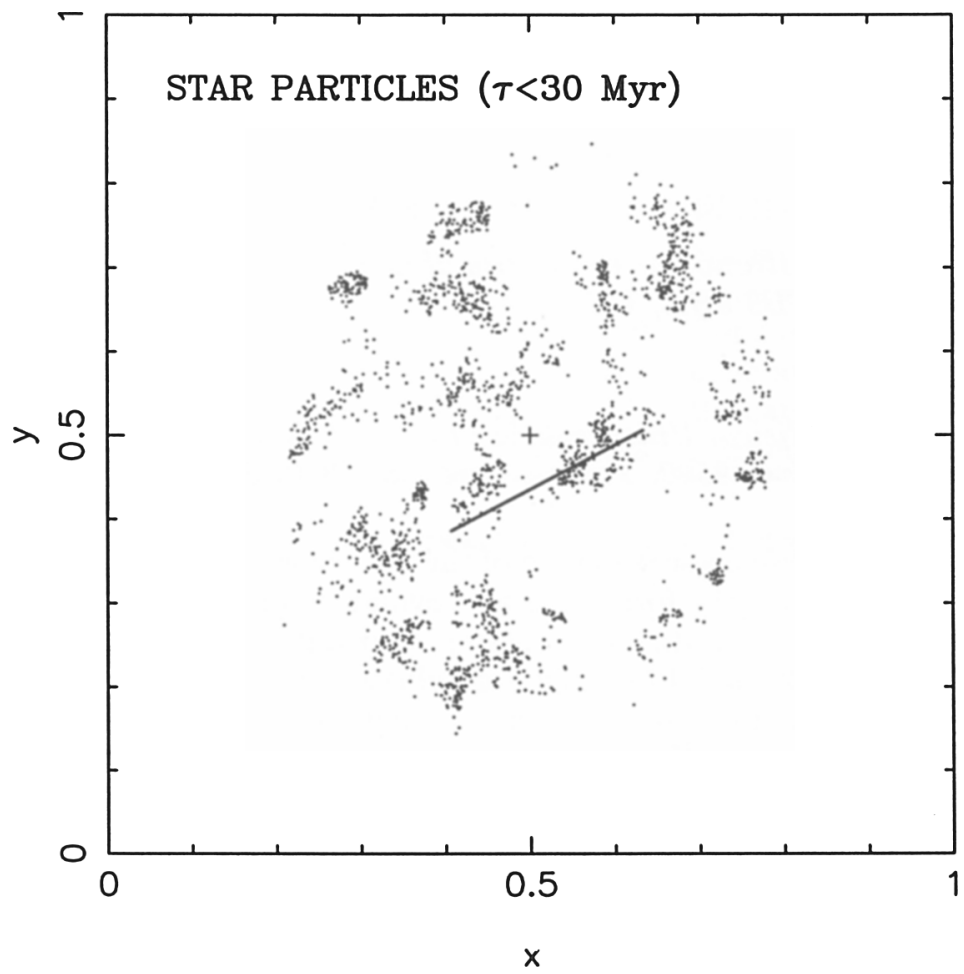

Figure 1. The star particle (age less than $30 \mathrm{Myr}$ ) distribution generated by the model at $T=300 \mathrm{Myr}$ after the start of the simulation. The disk center and the major axis of the bar are shown. Units are in tens of kpc.

simulation code incorporates a mechanism for propagating star formation, the large-scale spiral features are produced by the off-center bar potential (Colin \& Athanassoula 1989), not by the combination of propagating star formation plus shear. Nevertheless, the mechanism of propagating star formation is responsible for the generation of stellar complexes and gaseous structures up to $1 \mathrm{kpc}$ in size which are probably associated with the Shapley Constellations and supergiant shells. Refer to Gardiner et al. (1998b) for a more detailed account of this work.

\section{References}

Colin, J., \& Athanassoula, E. 1989, A\&A, 214, 99

Dottori, H., Bica, E., Claria, J.J., \& Puerari, I. 1996, ApJ, 461, 742

Gardiner, L.T, Turfus, C., \& Wang, M. 1998a, PASJ, 50, 375

Gardiner, L.T, Turfus, C., \& Putman, M.E. 1998b, ApJ, 507, L35

Smith, A.M., Cornett R.H., \& Hill R.S. 1987, ApJ, 320, 609 